\title{
Investigating Local Corrosion Processes in Real and Diffraction Space by in situ TEM Liquid Cell Experiments
}

\author{
Jordan Key ${ }^{1}$, Shixiang Zhu², Christopher M. Rouleau ${ }^{3}$, Raymond R. Unocic ${ }^{3}$, Yao Xie², Josh Kacher ${ }^{1}$ \\ 1. Georgia Institute of Technology, Materials Science and Engineering Department, Atlanta, GA, USA \\ 2. Georgia Institute of Technology, Industrial and Systems Engineering Department, Atlanta, GA, USA \\ 3. Oak Ridge National Laboratory, Center for Nanophase Materials Sciences, Oak Ridge, TN, USA
}

The development of liquid cell TEM holders and advances in electron detection technology have extended investigations of Processing-Structure-Property relations to aqueous environments within an electron microscope. In situ liquid cell microscopy allows for the direct observation of active corrosion events in real time (100 frames per second) and at nanometer spatial resolution [1,2]. This provides a wealth of information on localized corrosion mechanisms and kinetics, corrosion front morphology (including fractality), and phase transformations [3]. These measurements generate large data sets and require the application of sophisticated image recognition and data science methods for detailed analysis. The following work investigated the aqueous corrosion of sputtered iron thin films by acetic acid using in situ liquid cell microscopy. It demonstrates the strength of automated image analysis coupled with high framerate imaging during in situ TEM corrosion for directly correlating microstructural features with corrosion susceptibility.

Ferrous alloys have many applications that see exposure to corrosive environments, such as in the gas and oil industry. In this work, pure iron in $20 \mathrm{vol} \%$ aqueous acetic acid is taken as a model system. Iron thin films, with a thickness $\sim 100 \mathrm{~nm}$, were sputtered at room temperature onto the silicon nitride membrane of a liquid cell microchip device. The samples were then loaded into a commercial liquid cell holder (Protochips Inc.) and the corrosion processes were investigated using a FEI Titan operated at $300 \mathrm{kV}$. All images were collected using a Gatan OneView camera. Videos of the corrosion behavior were collected in real space with the TEM operating in bright field mode (Fig. 1) and in diffraction space with selected area diffraction patterns collected (Fig. 2). The grain size was on the order of 10-20 nm and the film was uniform. Electron energy-loss spectroscopy (EELS) was performed to ensure there was no oxidation of the films prior to the experiments.

Figure 1 is a time sequence showing beam-accelerated corrosion of the iron film. As can be seen, the corrosion front expands heterogeneously, consuming tens of grains, repassivating, and reinitiating at a different location. The reinitiation point could not be determined a priori. Additionally, at higher magnification a single field-of-view was maintained as the corrosion front passed. These observations behind the corrosion front also give insight into the corrosion mechanisms. Video of the corrosion front at a higher magnification show that propagation of the corrosion front occurs by discrete jumps (Fig. 3ab). By taking an image difference of two frames, the newly corroded region could be isolated and, by filtering and thresholding the difference image, the exact area of the corroded region could be determined (Fig. 3b-c). In this case, $180 \mathrm{~nm}^{2}$ was consumed by one discrete jump of the corrosion front, correlating to a spherical region with diameter approximately equal to $15 \mathrm{~nm}$. The similarity in size of the corroded region and the average grain size suggests that the front propagation behavior is influenced by the film microstructure. Image analysis of the diffraction patterns (Fig. 2) shows that the film is initially composed of iron with a random texture. As the corrosion process progressed, the sharpness of the diffraction ring decreased, suggesting a reduction in the crystalline area; this is readily apparent from the loss of higher- 
order ring patterns in Figure 2b. Signal detection algorithms were used to isolate diffraction peaks in the patterns and individual peaks were detected that matched the expected spacing for $\mathrm{Fe}_{2} \mathrm{O}_{3}$ prior to total dissolution of the film. Using thousands of diffraction patterns, crystallographic evolution can be tracked in time or in space to extract information that is difficult to obtain by visual inspection. Such rapid, automated image analysis of the data proves to be a powerful way to extract information from large data sets. Future work will seek to more closely integrate the real-space and diffraction imaging for correlated analysis of the crystallographic and morphological features of corrosion behavior [4].

[1] S.W. Chee et al, Chem. Commun. 51 (2015), p. 168.

[2] D. Gross et al, MS\&T Proceedings (2016).

[3] C. Vautrin-Ul et al, Corrosion Science 50 (2008), p. 2149.

[4] JK, SZ, YX, and JK acknowledge support from the Institute of Materials at Georgia Tech. Fe sputtering and in situ liquid cell microscopy experiments were supported as part of a user proposal at Oak Ridge National Laboratory's Center for Nanophase Materials Sciences (CNMS), which is a U.S. Department of Energy, Office of Science User Facility (CR and RU).
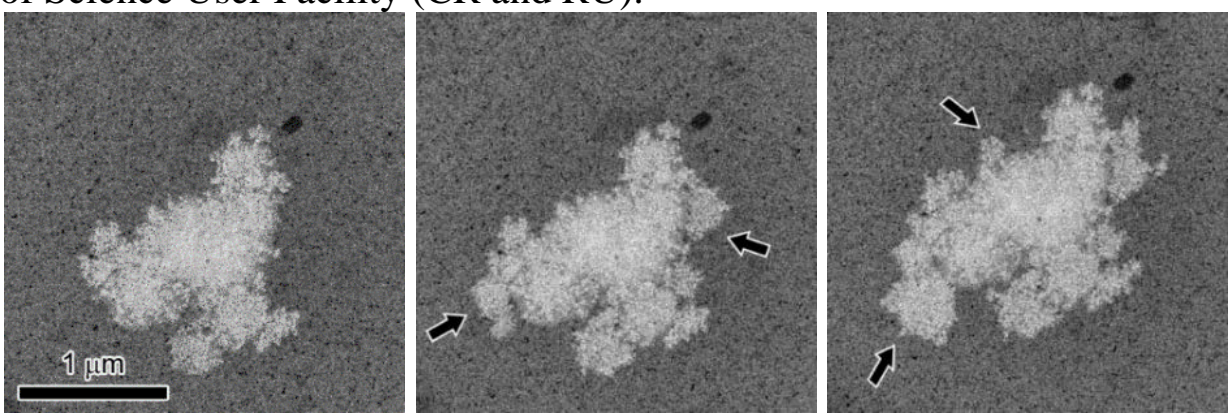

Figure 1. Time sequence from bright field video of a corrosion event at $t=0 \mathrm{~s}, 10 \mathrm{~s}$, and $28 \mathrm{~s}$, respectively, showing propagation of the corrosion front.
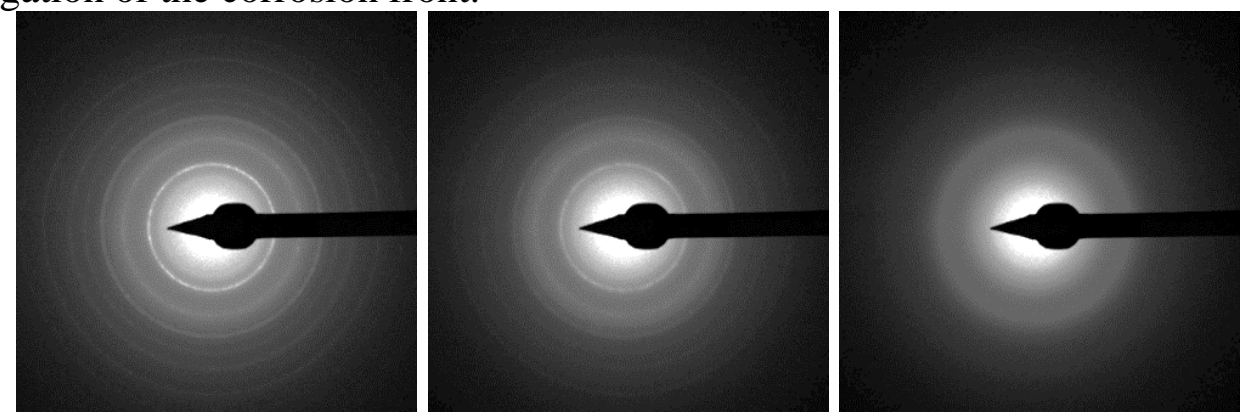

Figure 2. Time sequence of diffraction patterns at $t=23 \mathrm{~s}, 26 \mathrm{~s}$, and 33s, respectively, showing phase change at one location as the corrosion proceeds.

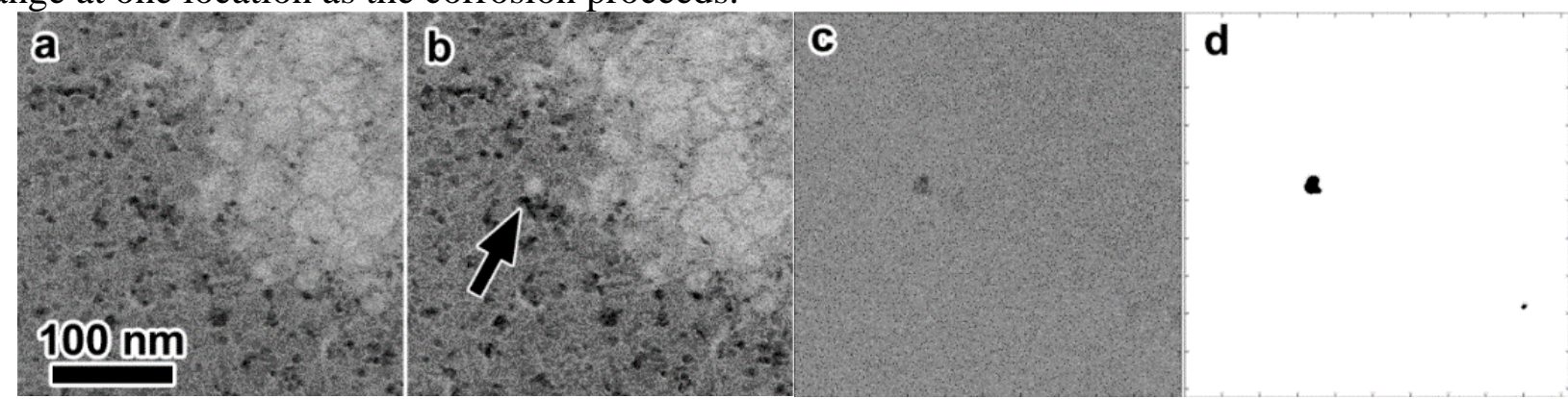

Figure 3. Image analysis of discrete jump event during the corrosion front propagation: a) prior to discrete jump event, b) highlighting the jump, c) image difference, and d) thresholding of image difference. 